\title{
Need Assessment for Smartphone-Based Cardiac Telerehabilitation
}

\author{
Ji-Su Kim, RN, MSN ${ }^{1}$, Doeun Yun, RN, BSN ${ }^{1}$, Hyun Joo Kim, $\mathrm{PT}^{2}$, Ho-Youl Ryu, RN, MSN ${ }^{1}$, \\ Jaewon Oh, MD, $\mathrm{PhD}^{3}$, Seok-Min Kang, $\mathrm{MD}, \mathrm{PhD}^{3}$ \\ ${ }^{1}$ Cardiac Wellness Center, ${ }^{2}$ Cardiovascular Laboratory Part, Severance Hospital, Seoul, Korea; ${ }^{3}$ Division of Cardiology, Severance Cardiovascular Hospital and \\ Cardiovascular Research Institute, Yonsei University College of Medicine, Seoul, Korea
}

Objectives: To identify the current status of smartphone usage and to describe the needs for smartphone-based cardiac telerehabilitation of cardiac patients. Methods: In 2016, a questionnaire survey was conducted in a supervised ambulatory cardiac rehabilitation (CR) program in a university affiliated hospital with the participation of heart failure or heart transplantation patients who were smartphone users. The questionnaire included questions regarding smartphone usage, demands for smartphone-based disease education, and home health monitoring systems. Results were described and analyzed according to principal diagnosis. Results: Ninety-six patients (66\% male; mean age, $53 \pm 11$ years), including 56 heart failure and 40 heart transplantation patients, completed the survey (completion rate, 95\%). The median daily smartphone usage time was 120 minutes (interquartile range, 60-300), and the most frequently used smartphone function was text messaging (61.5\%). Of the patients, $26 \%$ stated that they searched for health-related information using their smartphones more than 1 time per week. The major source of health-related information was Internet browsing (50.0\%), and the least sought source was the hospital's website (3.1\%). Patients with heart failure expressed significantly higher needs for disease education on treatment plan, home health monitoring of blood pressure, and body weight $\left(\chi^{2}=5.79,6.27,4.50, p<0.05\right)$. Heart transplantation patients expressed a significant need for home health monitoring of body temperature $\left(\chi^{2}=5.25, p<0.05\right)$. Conclusions: Heart failure and heart transplantation patients show high usage of and interest in mobile health technology. A smartphone-based cardiac telerehabilitation program should be developed based on high demand areas and modified to suit to each principal diagnosis.

Keywords: Telerehabilitation, Cardiac Rehabilitation, Needs Assessment, Smartphone, Mobile Applications

Submitted: July 12, 2018

Revised: 1st, August 29, 2018; 2nd, October 10, 2018

Accepted: October 24, 2018

\section{Corresponding Author}

Seok-Min Kang, MD, PhD

Division of Cardiology, Severance Cardiovascular Hospital and Cardiovascular Research Institute, Yonsei University College of Medicine, 50-1 Yonsei-ro, Seodaemun-gu, Seoul 03722, Korea. Tel: +822-2228-8450, E-mail: smkang@yuhs.ac (https://orcid.org/00000001-9343-952X)

This is an Open Access article distributed under the terms of the Creative Commons Attribution Non-Commercial License (http://creativecommons.org/licenses/by$\mathrm{nc} / 4.0 /$ ) which permits unrestricted non-commercial use, distribution, and reproduction in any medium, provided the original work is properly cited.

(c) 2018 The Korean Society of Medical Informatics

\section{Introduction}

Patients with heart disease have diverse risks associated with their lifestyle. Therefore, there is a need to enhancing their motivation to adhere to an appropriate lifestyle through the acquisition of knowledge about disease management and lifestyle improvement to prevent the recurrence of disease after discharge. Cardiac rehabilitation (CR) is a multidisciplinary program including education for risk factors; it significantly reduces the mortality of patients with myocardial infarction, ischemic heart disease, and heart failure $[1,2]$. In addition, CR reduces the burden on the healthcare system by maximizing the physical, psychological, and social condition of patients and encouraging behavior that can reduce or 
prevent further events $[3,4]$. Although CR may improve the mortality and morbidity rates of heart disease, low utilization remains a problem to be solved. Globally, CR participation rates are low, ranging from $14 \%$ to $43 \%$, with high dropout rates after enrollment [5]. Factors associated with participation in CR include patient, medical, and healthcare-oriented characteristics, as well as availability, affordability, and accessibility of a program, and the limitation of a participant's time and location in relation to a CR center are considered major factors that can negatively affect CR participation [6]. To overcome these barriers, cardiac telerehabilitation was introduced based on the use of smartphone [7].

Mobile health (mHealth) applications and cardiac telerehabilitation have developed fast and have potential for cardiovascular disease prevention and management $[7,8]$. mHealth is defined as healthcare supported by mobile devices, such as mobile phones, patient monitoring devices, interactive websites, and other wireless devices to support health objectives and behavior change $[9,10]$. Mobile phone ownership was reported to be $95 \%$ in the United States; in particular, smartphone ownership was $77 \%$ among adults in 2018 [11]. Currently, about 2 billion people own smartphones, which is an increase of $42 \%$ in comparison to 2011 [12]. South Korea has had the highest smartphone ownership among all countries, and smartphone penetration reached 88\% in 2016 [13].

Mobile technology has advanced rapidly, along with the popularization of smartphones, while various software applications have been developed. The introduction of various types of applications provides enormous opportunities for the delivery of healthcare services. Personalized care based on the characteristics of the individual has become possible, thereby enhancing the efficiency of such services [14]. Moreover, by enabling communication between providers and patients regardless of spatio-temporal limitations, more people can provide necessary care, leading to higher satisfaction than that for conventional interventions [15]. Recently, using monitoring sensors and sending feedback to providers and patients has provided new areas of treatment and development. Technology for engaging in and monitoring physical activity is becoming more readily available and can affect current CR provision. Some evidence has shown that cardiac telerehabilitation as a solution for cardiac patients is more feasible and effective than conventional center-based CR [16]. Various mHealth and telerehabilitation services have been developed and used so far, but more research and development is needed [7]. Furthermore, there are still major difficulties in integrating mHealth into daily clinical practice. One problem is that the development of mHealth is focused on the technological part, not on the patient's needs and expectations to use the technology [8].

It is believed that developing and applying programs that meet the interests and needs of the patients are important. A pilot study evaluated the phone usage and needs of patients with coronary artery diseases with regards to an mHealth healthy eating program [17]. It found that mobile phone usage was high among participants, and the participants were interested in receiving the program through mobile technology. Another study documented patients' current technology usage, interests in, and preferences for telerehabilitation, and their relationship with age, gender, and other characteristics [18]. Although the characteristics and preferences of telerehabilitation subjects were investigated through those studies, it differences between diagnoses should be identified. A previously developed app called AnSim (Hanmi Healthcare, Seoul, Korea) for cardiac patients, specifically focuses on coronary artery disease patients after percutaneous coronary intervention. CR programs are offered to patients with various cardiac diseases. Specified disease management is required for patients with coronary artery disease, post-operation and after heart failure (HF) or after heart transplantation (HT) according to their diagnosis. In particular, HF is a subtle and complex disease. Because HF patients have a high risk of an arrhythmic event, they require more attention, even in center-based CR programs [1]. If a smartphone-based cardiac telerehabilitation program could be tailored to each diagnosis, it would be able to provide more effective and efficient management.

This study aimed to identify the current smartphone usage status of selected patients with HF and HT and to describe their needs for smartphone-based disease education through a home health monitoring system.

\section{Methods}

\section{Study Design}

This cross-sectional survey was designed to investigate the needs of Korean cardiac patients for smartphone-based disease education and home health monitoring systems as part of a study to develop a cardiac telerehabilitation smartphone application. The study protocol was approved by the Institutional Review Board of Severance Hospital (No. 1-20160044). All patients gave informed consent.

\section{Participants}

We recruited eligible participants who had been diagnosed with heart failure and/or had undergone heart transplan- 
tation. All of them could perform exercises and owned a smartphone. Only those who followed the intervention using their smartphone or used at least some of the applications were included. Participants were recruited from a supervised ambulatory CR program in a university-affiliated hospital in Seoul from September 30 to December 5, 2016. A researcher assisted them if they had problems in completing the questionnaires. The participants required approximately 20 to 25 minutes to finish the questionnaire. Of 101 participants, 96 patients completed this study (completion rate, 95\%).

\section{Measurements}

Participants completed the self-report questionnaire, which assessed their experience of participating in disease education programs, their smartphone usage, and their needs for smartphone-based disease education and home health monitoring systems.

The questionnaire used in the survey included 12 questions, of which there were 10 close-ended response items and 2 open-ended questions. There were 6 questions on their experience with cardiac disease-related education, its venue, type, medium, effects, and their needs for education on cardiac diseases; 4 questions on their average hours of smartphone use, type of functions used, frequency of searching for health-related information using a smartphone, and the searching channel for health-related information to identify the current status of smartphone usage; and 2 questions on their needs for smartphone-based disease education and home health monitoring systems. The questions of the survey were developed by the researchers.

Participants' weight, height, heart rate, blood pressure, and cardiopulmonary capacity determined through a cardiopulmonary exercise test were measured before the survey was conducted. Socio-demographic data (age, gender, education level, residence, marital status, employment status, and health behavior) were collected after the survey. The medical records were reviewed to identify clinical characteristics, including comorbidity, experience of procedures/surgeries, medications, and left ventricle ejection fraction.

\section{Data Analysis}

All statistical analyses were conducted using SPSS Statistical Software 23.0 versions for Windows (IBM Corporation, Armonk, NY, USA). Analysis of the survey responses was largely descriptive. Descriptive statistics, such as means, standard deviations (SD), medians, interquartile range (IQR), frequencies, and percentages, were used to describe the demographic and clinical characteristics of the participants.
Comparisons of differences between diagnosis groups were made using a $t$-test for continuous variables and a chi-square test for categorical variables. Questionnaires were excluded from the analysis when 6 or more answers were missing. Statistical significance was set at $p<0.05$.

\section{Results}

\section{Demographics}

The general characteristics of the respondents are presented in Table 1. The mean age of the participants was $53 \pm 11$ years (range, $20-80$ years), and 63 patients (65.6\%) were men. The distribution of respondents by age was the following: $20-29$ (2.1\%), 30-39 (9.4\%), 40-49 (24.0\%), 50-59 (34.4\%), 60-69 (26.0\%), 70-79 (3.1\%), and over 80 years (1.0\%). Patients included of HF patients (58.3\%) and HT patients (41.7\%). The HT patients' body mass index (BMI) values were significantly lower than those of the HF patients (mean, $24.2 \pm 2.7$ vs. $27.0 \pm 5.2 \mathrm{~kg} / \mathrm{m}^{2} ; p=0.001$ ). There were significant differences regarding some of the comorbidities and experiences of procedures/surgeries according to diagnosis. The left ventricular ejection fractions of HF patients were significantly lower than those of HT patients $(p<$ $0.01)$. There were no significant differences regarding sociodemographics between diagnoses.

\section{Patients' Experience of Prior Heart Disease Education Programs}

Of the 96 participants, 42 (43.8\%) had participated in disease education programs before this study, and $95.2 \%$ had attended an education program in the hospital. The education program included topics such as nutrition (83.3\%), exercise and disease management (71.4\%), and medication (61.9\%). Over half of the education programs used a booklet as the medium for education (54.8\%). Over $90 \%$ of patients reported that the prior education program was useful to manage their disease. No significant differences were found in participation rates of disease education programs and the needs for disease education according to diagnosis or general and disease-related characteristics.

\section{Current Smartphone Usage}

Table 2 shows the smartphone usage according to diagnosis. Median daily smartphone usage time was 120 minutes (IQR, 60-300 minutes) with no significant differences between diagnoses. The smartphone functions used most frequently were text messages (61.4\%), calls (54.2\%), and Internet browsing $(46.9 \%)$. Of the patients, $26 \%$ stated that they 
Table 1. Demographic characteristics according to principal diagnosis

\begin{tabular}{|c|c|c|c|c|c|}
\hline & All $(n=96)$ & $H F(n=56)$ & HT $(n=40)$ & $\chi^{2} / t$ & $p$-value \\
\hline Gender, male & $63(65.6)$ & $37(66.1)$ & $26(65.0)$ & 0.012 & 0.913 \\
\hline Age (yr) & $52.7 \pm 11.2$ & $52.4 \pm 12.6$ & $53.2 \pm 9.0$ & & \\
\hline $20-49$ & $34(35.4)$ & $20(35.8)$ & $14(35.0)$ & 0.365 & 0.833 \\
\hline $50-59$ & $33(34.4)$ & $18(32.1)$ & $15(37.5)$ & & \\
\hline $60-89$ & $29(30.2)$ & $18(32.1)$ & $11(27.5)$ & & \\
\hline \multicolumn{6}{|l|}{ Education level } \\
\hline Non-completed high school & $9(9.4)$ & $5(8.9)$ & $4(10.0)$ & 0.640 & 0.726 \\
\hline Completed high school & $43(44.8)$ & $27(48.2)$ & $16(40.0)$ & & \\
\hline$\geq$ College & $44(45.8)$ & $24(42.9)$ & $20(50.0)$ & & \\
\hline Residence, metropolitan & $73(76.0)$ & $45(80.4)$ & $28(70.0)$ & 1.374 & 0.241 \\
\hline Spouse, married/partnered & $64(66.7)$ & $35(62.5)$ & $29(72.5)$ & 1.050 & 0.306 \\
\hline Currently employed, yes & $50(52.1)$ & $29(51.8)$ & $21(52.5)$ & 0.005 & 0.945 \\
\hline Smoking, yes & $10(10.4)$ & $7(12.5)$ & $3(7.5)$ & 0.625 & 0.429 \\
\hline Drinking alcohol ${ }^{\mathrm{a}}$, yes & $31(32.3)$ & $20(35.7)$ & $11(27.5)$ & 0.720 & 0.396 \\
\hline Regular exercise, yes & $56(58.3)$ & $36(64.3)$ & $20(50.0)$ & 1.959 & 0.162 \\
\hline \multicolumn{6}{|l|}{ Medication adherence ${ }^{b}$} \\
\hline Motivation & $2.2 \pm 0.8$ & $2.2 \pm 0.9$ & $2.3 \pm 0.7$ & -0.986 & 0.327 \\
\hline Knowledge & $2.2 \pm 0.6$ & $2.2 \pm 0.6$ & $2.2 \pm 0.7$ & 0.205 & 0.838 \\
\hline Total & $4.4 \pm 1.1$ & $4.3 \pm 1.1$ & $4.5 \pm 1.1$ & -0.608 & 0.545 \\
\hline BMI $\left(\mathrm{kg} / \mathrm{m}^{2}\right)$ & $25.8 \pm 4.6$ & $27.0 \pm 5.2$ & $24.2 \pm 2.7$ & 3.473 & 0.001 \\
\hline Under/normal weight $(<23)$ & $25(26.0)$ & $12(21.4)$ & $13(32.5)$ & & \\
\hline Overweight (23-24.9) & $18(18.8)$ & $6(10.8)$ & $12(30.0)$ & & \\
\hline Mild obesity (25-29.9) & $39(40.6)$ & $26(46.4)$ & $13(32.5)$ & & \\
\hline Obesity $(>30)$ & $14(14.6)$ & $12(21.4)$ & $2(5.0)$ & & \\
\hline Systolic BP (mmHg) & $124.3 \pm 17.2$ & $126.5 \pm 17.9$ & $121.3 \pm 16.0$ & 1.490 & 0.140 \\
\hline Diastolic BP (mmHg) & $81.6 \pm 12.6$ & $83.0 \pm 13.2$ & $79.6 \pm 11.6$ & 1.310 & 0.193 \\
\hline Heart rate (bpm) & $81.5 \pm 14.5$ & $79.2 \pm 12.5$ & $84.7 \pm 16.6$ & -1.771 & 0.081 \\
\hline LV ejection fraction (\%) & $53.0 \pm 14.8$ & $48.5 \pm 15.9$ & $57.6 \pm 12.2$ & -2.829 & 0.006 \\
\hline LV ejection fraction, $\leq 45$ & $22(22.9)$ & $17(30.4)$ & $5(12.5)$ & 8.734 & 0.003 \\
\hline Duration of illness (yr) & $3.7 \pm 4.1$ & $3.5 \pm 4.1$ & $4.1 \pm 4.0$ & -0.708 & 0.481 \\
\hline \multicolumn{6}{|l|}{ Comorbid disease, yes } \\
\hline Hypertension & $62(64.6)$ & $41(73.2)$ & $21(52.5)$ & 4.377 & 0.036 \\
\hline Diabetes & $19(19.8)$ & $5(8.9)$ & $14(35.0)$ & 9.991 & 0.002 \\
\hline Chronic kidney disease & $6(6.3)$ & $3(5.4)$ & $3(7.5)$ & 0.183 & 0.669 \\
\hline \multicolumn{6}{|c|}{ Experience of procedure/surgery, yes } \\
\hline $\mathrm{PCI} / \mathrm{CABG}$ & $11(11.4)$ & $2(3.6)$ & $9(22.5)$ & 8.240 & 0.004 \\
\hline Valve surgery & $9(9.4)$ & $2(3.6)$ & $7(17.5)$ & 5.328 & 0.021 \\
\hline ICD/CRT-D & $6(6.2)$ & $1(1.8)$ & $5(12.5)$ & 4.571 & 0.033 \\
\hline \multicolumn{6}{|l|}{ CPET } \\
\hline Peak $\mathrm{VO}_{2}(\mathrm{~mL} / \mathrm{kg} / \mathrm{min})$ & $23.8 \pm 6.4$ & $24.2 \pm 6.3$ & $23.1 \pm 6.4$ & 0.813 & 0.419 \\
\hline Test duration (min) & $774.0 \pm 185.7$ & $776.7 \pm 179.5$ & $771.8 \pm 199.1$ & 0.124 & 0.902 \\
\hline Peak RER & $1.1 \pm 0.1$ & $1.1 \pm 0.1$ & $1.1 \pm 0.1$ & -0.013 & 0.990 \\
\hline
\end{tabular}


Table 1. Continued

\begin{tabular}{lccccc}
\hline & All $(\mathrm{n}=96)$ & HF $(\mathrm{n}=56)$ & HT $(\mathrm{n}=40)$ & $\chi^{2} / t$ & $p$-value \\
\hline $\begin{array}{l}\text { Previous experience of participation in } \\
\text { education program, yes }\end{array}$ & $42(43.8)$ & $26(46.4)$ & $16(40.0)$ & 0.392 & 0.531 \\
\hline
\end{tabular}

Values are reported as number (\%) or mean \pm standard deviation.

HF: heart failure, HT: heart transplantation, BMI: body mass index, BP: blood pressure, LV: left ventricle, PCI: percutaneous coronary intervention, CABG: coronary artery bypass graft surgery, ICD: implantable cardioverter defibrillator, CRT-D: cardiac resynchronization therapy devices, CPET: cardio-pulmonary exercise test, $\mathrm{VO}_{2}$ : peak oxygen uptake, RER: respiratory exchange ratio.

${ }^{a}$ Drinking alcohol is defined as consumption of more than 7 units per week.

${ }^{b}$ Medication adherence was measured by the Modified Morisky Scale.

Table 2. Usage of smartphones

\begin{tabular}{|c|c|c|c|c|c|}
\hline & All $(n=96)$ & $\mathrm{HF}(\mathrm{n}=56)$ & $\mathrm{HT}(\mathrm{n}=40)$ & $\chi^{2} / t$ & $p$-value \\
\hline Usage time (min/day) & $120(60-300)$ & $150(75-300)$ & $90(60-270)$ & 1.598 & 0.113 \\
\hline \multicolumn{6}{|l|}{ Frequent used function ${ }^{\mathrm{a}}$ (yes) } \\
\hline Text message & $59(61.5)$ & $38(67.9)$ & $21(52.5)$ & 2.323 & 0.127 \\
\hline Phone call & $52(54.2)$ & $33(58.9)$ & $19(47.5)$ & 1.228 & 0.268 \\
\hline Internet browsing & $45(46.9)$ & $25(44.6)$ & $20(50.0)$ & 0.269 & 0.604 \\
\hline Video & $18(18.8)$ & $10(17.9)$ & $8(20.0)$ & 0.070 & 0.791 \\
\hline Game & $9(9.4)$ & $6(10.7)$ & $3(7.5)$ & 0.284 & 0.731 \\
\hline \multicolumn{6}{|c|}{$\begin{array}{l}\text { Frequency of searching health-related information } \\
\text { using smartphone }\end{array}$} \\
\hline More than 1 times/week & $38(39.6)$ & $28(50.0)$ & $10(25.0)$ & 6.098 & 0.014 \\
\hline \multicolumn{6}{|c|}{$\begin{array}{l}\text { Channel of acquiring health-related information } \\
\text { through smartphone (yes) }{ }^{\mathrm{a}}\end{array}$} \\
\hline Internet browsing & $48(50.0)$ & $29(51.8)$ & $19(47.5)$ & 0.171 & 0.679 \\
\hline News article & $19(19.8)$ & $12(21.4)$ & $7(17.5)$ & 0.227 & 0.634 \\
\hline Personal website & $14(14.6)$ & $9(16.1)$ & $5(12.5)$ & 0.239 & 0.625 \\
\hline Application & $8(8.3)$ & $5(8.9)$ & $3(7.5)$ & 0.062 & 0.803 \\
\hline Hospital's website & $3(3.1)$ & $2(3.6)$ & $1(2.5)$ & 0.008 & 0.766 \\
\hline \multicolumn{6}{|c|}{$\begin{array}{l}\text { Overall interest in smartphone-based cardiac } \\
\text { rehabilitation }\end{array}$} \\
\hline Very high interest & $58(60.5)$ & $33(58.9)$ & $25(62.5)$ & 1.672 & 0.433 \\
\hline High interest & $37(38.5)$ & $23(41.1)$ & $14(35.0)$ & & \\
\hline Low/No interest & $1(1.0)$ & - & $1(2.5)$ & & \\
\hline
\end{tabular}

Values are reported as median (interquartile range) or number (\%).

${ }^{a}$ Multiple responses were applicable.

searched for health-related information using their smartphones more than 1 time per week (50\% of HF patients and $25 \%$ of HT patients; $p<0.05)$. The high-smartphone-usage group showed greater frequency of searching for healthrelated information using smartphones in univariate analysis $\left(\chi^{2}=10.93, p<0.01\right)$.

The major channel of health-related information acquired through the smartphone was the internet browsing (50.0\%), and the least sought source was the hospital's website (3.1\%). The participants expressed very high interest (60.4\%) and high interest (38.5\%) in smartphone-based CR. 


\section{Patients' Interests and Preferences for Smartphone- based CR}

The needs for disease education through smartphone application are presented in Figure 1. Education regarding 'actions for emergency situations, treatment plans, responding to their symptoms, and exercise/physical activity' received the highest rating for inclusion in a smartphone-based educational application. HF patients expressed significantly higher needs for smartphone-based disease education regarding 'treatment plans' $\left(\chi^{2}=5.79, p<0.05\right)$.

The needs for home health monitoring systems through smartphone applications regarding the separate CR components are presented in Figure 2. Monitoring of 'exercise, blood pressure, and body composition' received the highest rating. Participants expressed significantly higher needs for home health monitoring of 'blood pressure' and 'body weight' in the HF group $\left(\chi^{2}=6.27,4.50, p<0.05\right)$ and 'body temperature' in the HT group $\left(\chi^{2}=5.25, p<0.05\right)$.

\section{Discussion}

This study documented the use of current technology by cardiac patients and their interest in smartphone-based CR. The significance of this study is that it identified the needs of participants regarding smartphone application-based disease education and home health monitoring systems.

Chow et al. [7] reported that, due to the recent increase in the number of smartphone users, smartphone applications have become an important tool in disease management. Accordingly, mHealth and cardiac telerehabilitation have been established as a new form of disease management and have advanced greatly $[9,15]$. Varnfield et al. [19] reported that, since applications can be easily built into smartphones, cardiac patients can use them at any time or place, and as a result, these applications can be effective tools for realizing mobile education and health management. Currently, many applications use monitoring sensors and provide feedback about the user's biological information. Healthcare providers are able to monitor patients' heart rate, blood pressure, and other health parameters $[20,21]$, and there is growing interest in how to remotely manage heart failure patients using monitoring sensors and mobile applications [22].

Patients in this study showed a high interest in both smart-

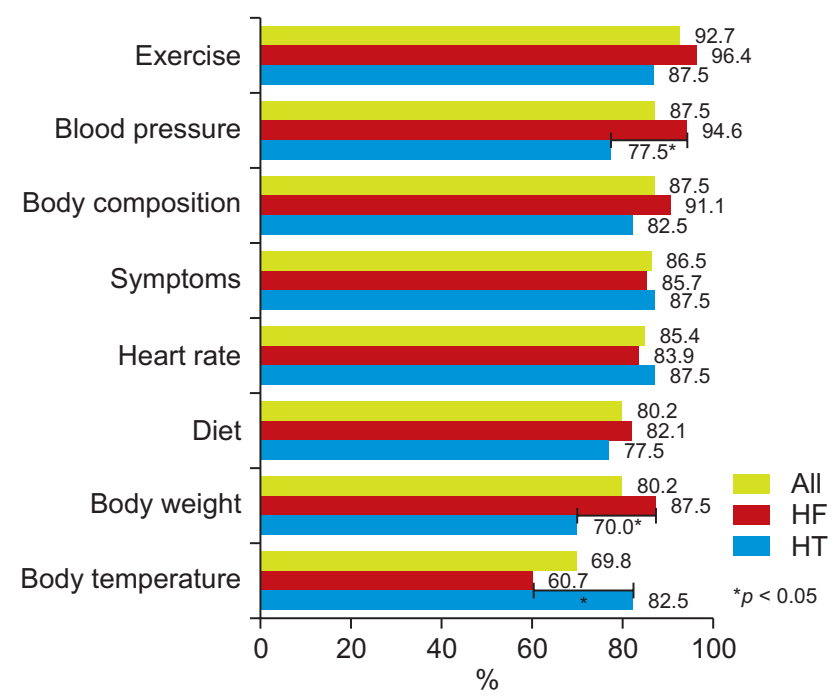

Figure 2. Needs for home health monitoring systems through smartphone applications. We identified the areas for which a home health monitoring system is needed through smartphone applications. Participants expressed significantly higher needs for home health monitoring of blood pressure and body weight in the HF group $\left(\chi^{2}=6.27,4.50, p<0.05\right)$ and body temperature in the HT group ( $\left.\chi^{2}=5.25, p<0.05\right)$. HF: heart failure, HT: heart transplantation.

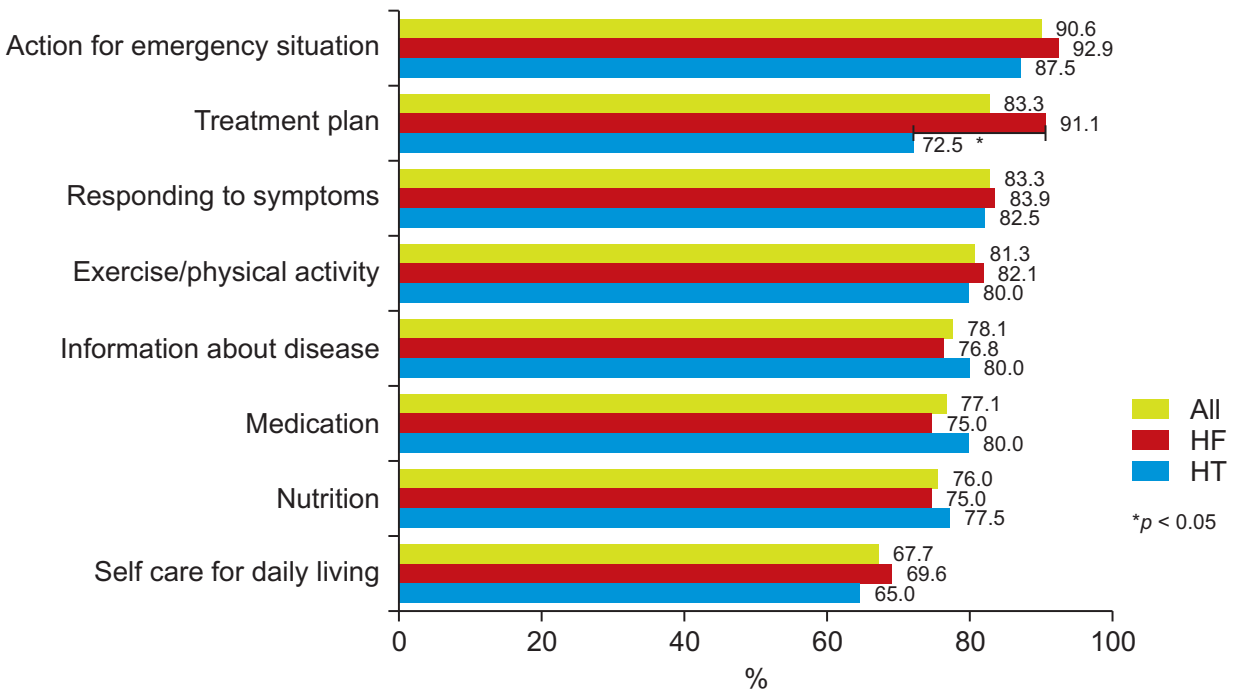

Figure 1. Needs for disease education through smartphone applications. The needs assessment of disease education through smartphone was conducted. HF patients expressed significantly higher needs for smartphonebased disease education for treatment plans $\left(\chi^{2}=5.79\right.$, $p<0.05)$. HF: heart failure, HT: heart transplantation. 
phone-based education and monitoring systems. One of the difficulties in applying mHealth is the lack of scientific evidence of efficiency [23]. Some interventions have been started to provide evidence to support mHealth's effectiveness, such as the Mobile Applications Rating Scale (MARS), a tool to assess the quality of health applications, and the United States Agency for International Development (USAID) site that collects evidence regarding mHealth [24,25]. The effectiveness of these interventions need to be verified to establish the confidence of both clinicians and patients and to facilitate engagement with these technologies. Moreover, inclusion of medical staff members in the development of smartphone-based cardiac telerehabilitation applications and active participation in patient care can be a solution to address the issues of the validity and reliability of these applications.

When participants search for health-related information through their smartphones, high rates $(50.0 \%)$ of internet browsing and low rates $(3.1 \%)$ of hospital website use were reported. On the Internet, there is a huge amount of information available; thus, the amount of inaccurate data is considerable [26]. Among these inaccurate data, healthcare providers should gather and provide high-quality healthrelated information for patients' healthcare. It is necessary to provide exact information without time and space limitations through validated smartphone-based CR applications.

Various heart diseases can progress to HF, and the end of treatment for HF is HT surgery [27]. In HT patients that have undergone a worsening HF process for a considerable period of time, although the left ventricle ejection fraction can be higher than in the HF group, the comorbidities can be higher and general condition can be lower than in HF patients. HF patients are treated to prevent progression of their disease, whereas the care of patients after HT is more focused on organ rejection and prevention of infection. Besides HF or HT, other heart diseases also have different focuses of disease management. For example, it was reported that monitoring of weight and symptoms was recommended to avoid hospital admission of HF patient [28]. In heart transplantation patients, taking of immunosuppressive medication is important as well as avoiding behaviors that may cause infection and monitoring the signs of infection [29]. Patients with coronary artery disease need to change their behavior to prevent further recurrent events [30]. Those previous study's results are in line with the findings of our study, showing high demands for home health monitoring of blood pressure and body weight in HF patients, while body temperature is of greatest importance for the HT group. The comparison of HF and HT conducted in this study showed that the needs for smartphone-based educational programs and home health monitoring systems were different for each disease. Also, the frequency of searching for health-related information using smartphones was higher in the HF group. This preference currently warrants a technology-based CR intervention tailored to the patient's needs and usage [8]. Smartphone-based CR has low participation of healthcare providers, but more enhanced accessibility and utility than center-based CR [16]. It is expected that smartphone-based $\mathrm{CR}$ can be developed in a patient-centric manner by reflecting the reported needs of the patients. Then higher participation of patients based on their needs may be facilitated.

This study had several limitations. First, our results were collected from a small group in a single center. Second, the sample was limited to people who had experienced heart failure and/or had undergone heart transplant surgery who participated in a phase II (outpatient) CR program. Consequently, the study could not identify any differences according to other cardiac disease, such as coronary artery or valvular diseases. We suggest further studies of diverse cardiac patients with a greater number of subjects.

In conclusion, the objective of this study was to provide basic information needed for the development of smartphone application-based disease education and home health monitoring systems for the management of heart diseases. This study documented a high usage rate and interest in mobile health technology among heart failure and post-hearttransplantation patients. Based on the demands and interest, a smartphone-based cardiac telerehabilitation program including disease education and an exercise program should be developed. This study found differences patients' needs regarding smartphone-based disease education and home health monitoring systems according to principal diagnosis. It will be necessary to consider both the generality of heart disease and the specificity of each principal diagnosis to develop and support the utilization of a smartphone-based cardiac telerehabilitation program.

\section{Conflict of Interest}

No potential conflict of interest relevant to this article was reported.

\section{Acknowledgments}

The study was funded by grants from the Ministry of Science and ICT, Korea (No. R7518-16-1021). 


\section{References}

1. Taylor RS, Sagar VA, Davies EJ, Briscoe S, Coats AJ, Dalal $\mathrm{H}$, et al. Exercise-based rehabilitation for heart failure. Cochrane Database Syst Rev 2014;(4):CD003331.

2. Sandercock G, Hurtado V, Cardoso F. Changes in cardiorespiratory fitness in cardiac rehabilitation patients: a meta-analysis. Int J Cardiol 2013;167(3):894-902.

3. Balady GJ, Williams MA, Ades PA, Bittner V, Comoss P, Foody JM, et al. Core components of cardiac rehabilitation/secondary prevention programs: 2007 update: a scientific statement from the American Heart Association Exercise, Cardiac Rehabilitation, and Prevention Committee, the Council on Clinical Cardiology; the Councils on Cardiovascular Nursing, Epidemiology and Prevention, and Nutrition, Physical Activity, and Metabolism; and the American Association of Cardiovascular and Pulmonary Rehabilitation. Circulation 2007;115(20):2675-82.

4. National Health Service in England. Commissioning guidance for rehabilitation [Internet]. London: NHS England; c2016 [cited at 2018 Apr 13]. Available from: https://www.england.nhs.uk/wp-content/uploads/2016/04/rehabilitation-comms-guid-16-17.pdf.

5. Bjarnason-Wehrens B, McGee H, Zwisler AD, Piepoli MF, Benzer W, Schmid JP, et al. Cardiac rehabilitation in Europe: results from the European Cardiac Rehabilitation Inventory Survey. Eur J Cardiovasc Prev Rehabil 2010;17(4):410-8.

6. Martin AM, Woods CB. What sustains long-term adherence to structured physical activity after a cardiac event? J Aging Phys Act 2012;20(2):135-47.

7. Chow CK, Ariyarathna N, Islam SM, Thiagalingam A, Redfern J. mHealth in cardiovascular health care. Heart Lung Circ 2016;25(8):802-7.

8. Saner H. e-Cardiology and e-Health: from industrydriven technical progress to clinical application. Eur J Prev Cardiol 2014;21(2 Suppl):2-3.

9. Yi JY, Kim Y, Cho YM, Kim H. Self-management of chronic conditions using mHealth interventions in Korea: a systematic review. Healthc Inform Res 2018;24(3):187-97.

10. World Health Organization. mHealth: new horizons for health through mobile technologies. Geneva, Switzerland: World Health Organization; 2011.

11. Pew Research Center. Mobile phone ownership over time [Internet]. Washington (DC): Pew Research Center; c2018 [cited at 2018 May 22]. Available from: http:// www.pewinternet.org/fact-sheet/mobile/.

12. Statista Inc. Smartphones industry: statistics and facts [Internet]. New York (NY): Statista Inc.; c2018 [cited at 2018 may 5]. Available from: https://www.statista.com/ topics/840/smartphones/.

13. Poushter J. Smartphone ownership rates skyrocket in many emerging economies, but digital divide remains [Internet]. Washington (DC): Pew Research Center; c2016 [cited at 2018 May 5]. Available from: http://www. pewglobal.org/2016/02/22/smartphone-ownershiprates-skyrocket-in-many-emerging-economies-butdigital-divide-remains/.

14. Burke LE, Ma J, Azar KM, Bennett GG, Peterson ED, Zheng Y, et al. Current science on consumer use of mobile health for cardiovascular disease prevention: a scientific statement from the American Heart Association. Circulation 2015;132(12):1157-213.

15. Han M, Lee E. Effectiveness of mobile health application use to improve health behavior changes: a systematic review of randomized controlled trials. Healthc Inform Res 2018;24(3):207-26.

16. Frederix I, Vanhees L, Dendale P, Goetschalckx K. A review of telerehabilitation for cardiac patients. J Telemed Telecare 2015;21(1):45-53.

17. Dale LP, Whittaker R, Eyles H, Mhurchu CN, Ball K, Smith N, et al. Cardiovascular disease self-management: pilot testing of an mHealth healthy eating program. J Pers Med 2014;4(1):88-101.

18. Buys R, Claes J, Walsh D, Cornelis N, Moran K, Budts W, et al. Cardiac patients show high interest in technology enabled cardiovascular rehabilitation. BMC Med Inform Decis Mak 2016;16:95.

19. Varnfield M, Karunanithi M, Lee CK, Honeyman E, Arnold D, Ding H, et al. Smartphone-based home care model improved use of cardiac rehabilitation in postmyocardial infarction patients: results from a randomized controlled trial. Heart 2014;100:1770-9.

20. Lowres N, Neubeck L, Salkeld G, Krass I, McLachlan AJ, Redfern J, et al. Feasibility and cost-effectiveness of stroke prevention through community screening for atrial fibrillation using iPhone ECG in pharmacies: the SEARCH-AF study. Thromb Haemost 2014;111(6):1167-76.

21. Redfern J, Usherwood T, Harris MF, Rodgers A, Hayman N, Panaretto K, et al. A randomised controlled trial of a consumer-focused e-health strategy for cardiovascular risk management in primary care: the Consumer Navigation of Electronic Cardiovascular Tools (CON- 
NECT) study protocol. BMJ Open 2014;4(2):e004523.

22. Walsh JA 3rd, Topol EJ, Steinhubl SR. Novel wireless devices for cardiac monitoring. Circulation 2014;130(7):573-81.

23. Georgsson M, Staggers N. Quantifying usability: an evaluation of a diabetes mHealth system on effectiveness, efficiency, and satisfaction metrics with associated user characteristics. J Am Med Inform Assoc 2016;23(1):5-11.

24. mHealth Evidence by K4Health [Internet]. Baltimore (MD): mhealthevidence.org; c2016 [cited at 2018 Apr 5]. Available from: https://www.mhealthevidence.org/.

25. Stoyanov SR, Hides L, Kavanagh DJ, Zelenko O, Tjondronegoro D, Mani M. Mobile app rating scale: a new tool for assessing the quality of health mobile apps. JMIR Mhealth Uhealth 2015;3(1):e27.

26. Korp P. Health on the Internet: implications for health promotion. Health Educ Res 2006;21(1):78-86.
27. Cleland JG, McDonagh T, Rigby AS, Yassin A, Whittaker T, Dargie HJ, et al. The national heart failure audit for England and Wales 2008-2009. Heart 2011;97(11):87686.

28. Inglis SC, Clark RA, Dierckx R, Prieto-Merino D, Cleland JG. Structured telephone support or non-invasive telemonitoring for patients with heart failure. Cochrane Database Syst Rev 2015;(10):CD007228.

29. Brocks Y, Zittermann A, Grisse D, Schmid-Ott G, Stock-Gießendanner S, Schulz U, et al. Adherence of heart transplant recipients to prescribed medication and recommended lifestyle habits. Prog Transplant 2017;27(2):160-6.

30. Peterson JC, Allegrante JP, Pirraglia PA, Robbins L, Lane $\mathrm{KP}$, Boschert KA, et al. Living with heart disease after angioplasty: a qualitative study of patients who have been successful or unsuccessful in multiple behavior change. Heart Lung 2010;39(2):105-15. 